\title{
$p$ Harmonic Measure in Simply Connected Domains
}

\author{
John L. Lewis*† \\ Department of Mathematics, University of Kentucky \\ Lexington, KY 40506-0027, USA \\ Kaj Nyström $\ddagger$ \\ Department of Mathematics, Umeå University \\ S-90187 Umeå, Sweden \\ Pietro Poggi-Corradini \\ Department of Mathematics, Cardwell Hall, Kansas State University \\ Manhattan, KS 66506, USA
}

October 28, 2018

\begin{abstract}
Let $\Omega$ be a bounded simply connected domain in the complex plane, $\mathbb{C}$. Let $N$ be a neighborhood of $\partial \Omega$, let $p$ be fixed, $1<p<\infty$, and let $\hat{u}$ be a positive weak solution to the $p$ Laplace equation in $\Omega \cap N$. Assume that $\hat{u}$ has zero boundary values on $\partial \Omega$ in the Sobolev sense and extend $\hat{u}$ to $N \backslash \Omega$ by putting $\hat{u} \equiv 0$ on $N \backslash \Omega$. Then there exists a positive finite Borel measure $\hat{\mu}$ on $\mathbb{C}$ with support contained in $\partial \Omega$ and such that

$$
\int|\nabla \hat{u}|^{p-2}\langle\nabla \hat{u}, \nabla \phi\rangle d A=-\int \phi d \hat{\mu}
$$

whenever $\phi \in C_{0}^{\infty}(N)$. If $p=2$ and if $\hat{u}$ is the Green function for $\Omega$ with pole at $x \in \Omega \backslash \bar{N}$ then the measure $\hat{\mu}$ coincides with harmonic measure at $x, \omega=\omega^{x}$, associated to the Laplace equation. In this paper we continue the studies in [BL05], [L06] by establishing new results, in simply connected domains, concerning the Hausdorff dimension of the support of the measure $\hat{\mu}$. In particular, we prove results, for $1<p<\infty, p \neq 2$, reminiscent of the famous result of Makarov [Mak85] concerning the Hausdorff dimension of the support of harmonic measure in simply connected domains.
\end{abstract}

2000 Mathematics Subject Classification. Primary 35J25, 35J70.

Keywords and phrases: harmonic function, harmonic measure, $p$ harmonic measure, $p$ harmonic function, simply connected domain, Hausdorff measure, Hausdorff dimension.

\footnotetext{
*email: john@ms.uky.edu

${ }^{\dagger}$ Lewis was partially supported by DMS-0552281.

‡email: kaj.nystrom@math.umu.se

$\S$ Nyström was partially supported by grant VR-70629701 from the Swedish research council VR.

؟email: pietro@math.ksu.edu
} 


\section{Introduction}

Let $\Omega \subset \mathbf{R}^{n}$ be a bounded domain and recall that the continuous Dirichlet problem for Laplace's equation in $\Omega$ can be stated as follows. Given a continuous function $f$ on $\partial \Omega$, find a harmonic function $u$ in $\Omega$ which is continuous in $\bar{\Omega}$, with $u=f$ on $\partial \Omega$. Although such a classical solution may not exist, it follows from a method of Perron-Wiener-Brelot that there is a unique bounded harmonic function $u$ with continuous boundary values equal to $f$, outside a set of capacity zero (logarithmic capacity for $n=2$ and Newtonian capacity for $n>2$ ). The maximum principle and Riesz representation theorem yield, for each $x \in \Omega$, the existence of a Borel measure $\omega^{x}$ with $\omega^{x}(\partial \Omega)=1$, and

$$
u(x)=\int_{\partial \Omega} f(y) d \omega^{x}(y) \quad \text { whenever } x \in \Omega .
$$

Then, $\omega=\omega^{x}$ is referred to as the harmonic measure at $x$ associated with the Laplace operator.

Let also $g=g(\cdot)=g(\cdot, x)$ be the Green function for $\Omega$ with pole at $x \in \Omega$ and extend $g$ to $\mathbf{R}^{n} \backslash \Omega$ by putting $g \equiv 0$ on $\mathbf{R}^{n} \backslash \Omega$. Then $\omega$ is the Riesz measure associated to $g$ in the sense that

$$
\int\langle\nabla g, \nabla \phi\rangle d x=-\int \phi d \omega \text { whenever } \phi \in C_{0}^{\infty}\left(\mathbf{R}^{n} \backslash\{x\}\right)
$$

We define the Hausdorff dimension of $\omega$, denoted H-dim $\omega$, by

H-dim $\omega=\inf \left\{\alpha:\right.$ there exists $E$ Borel $\subset \partial \Omega$ with $H^{\alpha}(E)=0$ and $\left.\omega(E)=\omega(\partial \Omega)\right\}$,

where $H^{\alpha}(E)$, for $\alpha \in \mathbf{R}_{+}$, is the $\alpha$-dimensional Hausdorff measure of $E$ defined below. In the past twenty years a number of remarkable results concerning H-dim $\omega$ have been established in planar domains, $\Omega \subset \mathbf{R}^{2}$. In particular, Carleson [C85] showed that H-dim $\omega=1$ when $\partial \Omega$ is a snowflake and that $\mathrm{H}$-dim $\omega \leq 1$ for any self similar Cantor set. Later Makarov [Mak85] proved that H-dim $\omega=1$ for any simply connected domain in the plane. Furthermore, Jones and Wolff [JW88] proved that H-dim $\omega \leq 1$ whenever $\Omega \subset \mathbf{R}^{2}$ and $\omega$ exists and Wolff [W93] strengthened [JW88] by showing that $\omega$ is concentrated on a set of s finite $H^{1}$-measure. We also mention results of Batakis [Ba96], Kaufmann-Wu [KW85], and Volberg [V93] who showed, for certain fractal domains and domains whose complements are Cantor sets, that

$$
\text { Hausdorff dimension of } \partial \Omega=\inf \left\{\alpha: H^{\alpha}(\partial \Omega)=0\right\}>\mathrm{H}-\operatorname{dim} \omega \text {. }
$$

Finally we note that higher dimensional results for the dimension of harmonic measure can be found in [Bo87], [W95], and [LVV05].

In [BL05] the first author, together with Bennewitz, started the study of the dimension of a measure, here referred to as $p$ harmonic measure, associated with a positive $p$ harmonic function which vanishes on the boundary of certain domains in the plane. The study in [BL05] was continued in [L06]. Let $\mathbb{C}$ denote the complex plane and let $d A$ be Lebesgue measure on $\mathbb{C}$. If $O \subset \mathbb{C}$ is open and $1 \leq q \leq \infty$, let $W^{1, q}(O)$ be the space of equivalence classes of functions $\hat{u}$ with distributional gradient $\nabla \hat{u}=\left(\hat{u}_{x}, \hat{u}_{y}\right)$, both of which are $q$ th power integrable on $O$. Let

$$
\|\hat{u}\|_{1, q}=\|\hat{u}\|_{q}+\|\nabla \hat{u}\|_{q}
$$


be the norm in $W^{1, q}(O)$ where $\|\cdot\|_{q}$ denotes the usual Lebesgue $q$ norm in $O$. Let $C_{0}^{\infty}(O)$ be infinitely differentiable functions with compact support in $O$ and let $W_{0}^{1, q}(O)$ be the closure of $C_{0}^{\infty}(O)$ in the norm of $W^{1, q}(O)$. Let $\Omega \subset \mathbb{C}$ be a simply connected domain and suppose that the boundary of $\Omega, \partial \Omega$, is bounded and non empty. Let $N$ be a neighborhood of $\partial \Omega, p$ fixed, $1<p<\infty$, and let $\hat{u}$ be a positive weak solution to the $p$ Laplace equation in $\Omega \cap N$. That is, $\hat{u} \in W^{1, p}(\Omega \cap N)$ and

$$
\int|\nabla \hat{u}|^{p-2}\langle\nabla \hat{u}, \nabla \theta\rangle d A=0
$$

whenever $\theta \in W_{0}^{1, p}(\Omega \cap N)$. Observe that if $\hat{u}$ is smooth and $\nabla \hat{u} \neq 0$ in $\Omega \cap N$, then $\nabla$. $\left(|\nabla \hat{u}|^{p-2} \nabla \hat{u}\right) \equiv 0$, in the classical sense, where $\nabla$. denotes divergence. We assume that $\hat{u}$ has zero boundary values on $\partial \Omega$ in the Sobolev sense. More specifically if $\zeta \in C_{0}^{\infty}(N)$, then $\hat{u} \zeta \in W_{0}^{1, p}(\Omega \cap N)$. Extend $\hat{u}$ to $N \backslash \Omega$ by putting $\hat{u} \equiv 0$ on $N \backslash \Omega$. Then $\hat{u} \in W^{1, p}(N)$ and it follows from (1.1), as in [HKM93], that there exists a positive finite Borel measure $\hat{\mu}$ on $\mathbb{C}$ with support contained in $\partial \Omega$ and the property that

$$
\int|\nabla \hat{u}|^{p-2}\langle\nabla \hat{u}, \nabla \phi\rangle d A=-\int \phi d \hat{\mu}
$$

whenever $\phi \in C_{0}^{\infty}(N)$. We note that if $\partial \Omega$ is smooth enough, then $d \hat{\mu}=\left.|\nabla \hat{u}|^{p-1} d H^{1}\right|_{\partial \Omega}$. Note that if $p=2$ and if $\hat{u}$ is the Green function for $\Omega$ with pole at $x \in \Omega$ then the measure $\hat{\mu}$ coincides with harmonic measure at $x, \omega=\omega^{x}$, introduced above. We refer to $\hat{\mu}$ as the $p$ harmonic measure associated to $\hat{u}$. In [BL05], [L06] the Hausdorff dimension of the $p$ harmonic measure $\hat{\mu}$ is studied for general $p, 1<p<\infty$, and to state results from [BL05], [L06] we next properly introduce the notions of Hausdorff measure and Hausdorff dimension. In particular, let points in the complex plan be denoted by $z=x+i y$ and put $B(z, r)=\{w \in \mathbb{C}:|w-z|<r\}$ whenever $z \in \mathbb{C}$ and $r>0$. Let $d(E, F)$ denote the distance between the sets $E, F \subset \mathbb{C}$. If $\lambda>0$ is a positive function on $\left(0, r_{0}\right)$ with $\lim _{r \rightarrow 0} \lambda(r)=0$ define $H^{\lambda}$ Hausdorff measure on $\mathbb{C}$ as follows: For fixed $0<\delta<r_{0}$ and $E \subseteq \mathbb{R}^{2}$, let $L(\delta)=\left\{B\left(z_{i}, r_{i}\right)\right\}$ be such that $E \subseteq \cup B\left(z_{i}, r_{i}\right)$ and $0<r_{i}<\delta, \quad i=1,2, \ldots$ Set

$$
\phi_{\delta}^{\lambda}(E)=\inf _{L(\delta)} \sum \lambda\left(r_{i}\right)
$$

Then

$$
H^{\lambda}(E)=\lim _{\delta \rightarrow 0} \phi_{\delta}^{\lambda}(E) .
$$

In case $\lambda(r)=r^{\alpha}$ we write $H^{\alpha}$ for $H^{\lambda}$. We now define the Hausdorff dimension of the measure $\hat{\mu}$ introduced in (1.2) as

$$
\mathrm{H}-\operatorname{dim} \hat{\mu}=\inf \left\{\alpha: \text { there exists } E \text { Borel } \subset \partial \Omega \text { with } H^{\alpha}(E)=0 \text { and } \hat{\mu}(E)=\hat{\mu}(\partial \Omega)\right\} \text {. }
$$

In [BL05] the first author, together with Bennewitz, proved the following theorem.

Theorem A. Let $\hat{u}, \hat{\mu}$, be as in (1.1), (1.2). If $\partial \Omega$ is a quasicircle, then $H$-dim $\hat{\mu} \leq 1$ for $2 \leq p<\infty$, while $H$-dim $\hat{\mu} \geq 1$ for $1<p \leq 2$. Moreover, if $\partial \Omega$ is the von Koch snowflake then strict inequality holds for H-dim $\hat{\mu}$. 
In [L06] the results in [BL05] were improved at the expense of assuming more about $\partial \Omega$. In particular, we refer to [L06] for the definition of a $k$ quasi-circle. The following theorem is proved in [L06].

Theorem B. Given $p, 1<p<\infty, p \neq 2$, there exists $k_{0}(p)>0$ such that if $\partial \Omega$ is a $k$ quasi-circle and $0<k<k_{0}(p)$, then

(a) $\hat{\mu}$ is concentrated on a set of $\sigma$ finite $H^{1}$ measure when $p>2$.

(b) There exists $A=A(p), 0<A(p)<\infty$, such that if $1<p<2$, then $\hat{\mu}$ is absolutely continuous with respect to Hausdorff measure defined relative to $\tilde{\lambda}$ where

$$
\tilde{\lambda}(r)=r \exp [A \sqrt{\log 1 / r \log \log \log 1 / r}], 0<r<10^{-6} .
$$

We note that Makarov in [Mak85] proved Theorem B for harmonic measure $\omega, p=2$, when $\Omega$ is simply connected. Moreover, in this case it suffices to take $A=6 \sqrt{(\sqrt{24}-3) / 5}$, see [HK07]. In this paper we continue the studies in [BL05] and [L06] and we prove the following theorem.

Theorem 1. Given $p, 1<p<\infty, p \neq 2$, let $\hat{u}, \hat{\mu}$ be as in (1.1), (1.2), and suppose $\Omega$ is simply connected. Put

$$
\lambda(r)=r \exp [A \sqrt{\log 1 / r \log \log 1 / r}], 0<r<10^{-6} .
$$

Then the following is true.

(a) If $p>2$, there exists $A=A(p) \leq-1$ such that $\hat{\mu}$ is concentrated on a set of $\sigma$ finite $H^{\lambda}$ measure.

(b) If $1<p<2$, there exists $A=A(p) \geq 1$, such that $\hat{\mu}$ is absolutely continuous with respect to $H^{\lambda}$.

Note that Theorem 1 and the definition of H-dim $\hat{\mu}$ imply the following corollary.

Corollary 1. Given $p, 1<p<\infty, p \neq 2$, let $\hat{u}, \hat{\mu}$ be as in (1.1), (1.2), and suppose $\Omega$ is simply connected. Then $H$-dim $\hat{\mu} \leq 1$ for $2 \leq p<\infty$, while $H$-dim $\hat{\mu} \geq 1$ for $1<p \leq 2$.

In Lemma 2.4, stated below, we first show that it is enough to to prove Theorem 1 for a specific $p$ harmonic function $\hat{u}$ satisfying the hypotheses. Thus, we choose $z_{0} \in \Omega$ and let $u$ be the $p$ capacitary functions for $D=\Omega \backslash \bar{B}\left(z_{0}, d\left(z_{0}, \partial \Omega\right) / 2\right)$. Then $u$ is $p$ harmonic in $D$ with continuous boundary values, $u \equiv 0$ on $\partial \Omega$ and $u \equiv 1$ on $\partial B\left(z_{0}, d\left(z_{0}, \partial \Omega\right) / 2\right)$. Furthermore, to prove Theorem 1, we build on the tools and techniques developed in [BL05]. In particular, as noted in [BL05, sec. 7, Closing Remarks, problem 5], given the tools in [BL05] the main difficulty in establishing Theorem 1 is to prove the following result. 
Theorem 2. Given $p, 1<p<\infty, p \neq 2$, let $u, D$ be as above. There exists $c_{1} \geq 1$, depending only on $p$, such that

$$
c_{1}^{-1} \frac{u(z)}{d(z, \partial \Omega)} \leq|\nabla u(z)| \leq c_{1} \frac{u(z)}{d(z, \partial \Omega)} \text {, whenever } z \in D
$$

In fact, most of our effort in this paper is devoted to proving Theorem 2. Armed with Theorem 2 we then use arguments from [BL05] and additional measure-theoretic arguments to prove Theorem 1. To further appreciate and understand the importance of the type of estimate we establish in Theorem 2, we note that this type of estimate is also crucial in the recent work by the first and second author on the boundary behaviour, regularity and free boundary regularity for $p$ harmonic functions, $p \neq 2,1<p<\infty$, in domains in $\mathbf{R}^{n}, n \geq 2$, which are Lipschitz or which are well approximated by Lipschitz domains in the Hausdorff distance sense, see [LN07,LN,LN08a,LN08b]. Moreover, Theorem 2 seems likely to be an important step when trying to solve several problems for $p$ harmonic functions and $p$ harmonic measure, in planar simply-connected domains previously only studied in the case $p=2$, i.e., for harmonic functions and harmonic measure. In particular, we refer to [BL05, sec. 7, Closing Remarks] and [L06, Closing Remarks] for discussions of open problems.

The rest of the paper is organized as follows. In section 2 we list some basic local results for a positive $p$ harmonic function vanishing on a portion of $\partial \Omega$. In section 3 we use these results to prove Theorem 1 under the assumption that Theorem 2 is valid. In sections 4 and 5 we then prove Theorem 2.

Finally the first author would like to thank Michel Zinsmeister for some helpful comments regarding the proof of $(4.16)$.

\section{Basic Estimates.}

In the sequel $c$ will denote a positive constant $\geq 1$ (not necessarily the same at each occurrence), which may depend only on $p$, unless otherwise stated. In general, $c\left(a_{1}, \ldots, a_{n}\right)$ denotes a positive constant $\geq 1$, which may depend only on $p, a_{1}, \ldots, a_{n}$, not necessarily the same at each occurrence. $C$ will denote an absolute constant. $A \approx B$ means that $A / B$ is bounded above and below by positive constants depending only on $p$. In this section, we will always assume that $\Omega$ is a bounded simply connected domain, $0<r<\operatorname{diam} \partial \Omega$ and $w \in \partial \Omega$. We begin by stating some interior and boundary estimates for $\tilde{u}$, a positive weak solution to the $p$ Laplacian in $B(w, 4 r) \cap \Omega$ with $\tilde{u} \equiv 0$ in the Sobolev sense on $\partial \Omega \cap B(w, 4 r)$. That is, $\tilde{u} \in W^{1, p}(B(w, 4 r) \cap \Omega)$ and (1.1) holds whenever $\theta \in W_{0}^{1, p}(B(w, 4 r) \cap \Omega)$. Also $\zeta \tilde{u} \in W_{0}^{1, p}(B(w, 4 r) \cap \Omega)$ whenever $\zeta \in C_{0}^{\infty}(B(w, 4 r))$. Extend $\tilde{u}$ to $B(w, 4 r)$ by putting $\tilde{u} \equiv 0$ on $B(w, 4 r) \backslash \Omega$. Then there exists a locally finite positive Borel measure $\tilde{\mu}$ with support $\subset B(w, 4 r) \cap \partial \Omega$ and for which (1.2) holds with $\hat{u}$ replaced by $\tilde{u}$ and $\phi \in C_{0}^{\infty}(B(w, 4 r))$. Let $\max _{B(z, s)} \tilde{u}, \min _{B(z, s)} \tilde{u}$ be the essential supremum and infimum of $\tilde{u}$ on $B(z, s)$ whenever $B(z, s) \subset B(w, 4 r)$. For references to proofs of Lemmas 2.1 - 2.3 (see [BL05]). 
Lemma 2.1. Fix $p, 1<p<\infty$, and let $\Omega, w, r, \tilde{u}$, be as above. Then

$$
c^{-1} r^{p-2} \int_{B(w, r / 2)}|\nabla \tilde{u}|^{p} d x \leq \max _{B(w, r)} \tilde{u}^{p} \leq c r^{-2} \int_{B(w, 2 r)} \tilde{u}^{p} d x .
$$

If $B(z, 2 s) \subset \Omega$, then

$$
\max _{B(z, s)} \tilde{u} \leq c \min _{B(z, s)} \tilde{u}
$$

Lemma 2.2. Let $p, \Omega, w, r, \tilde{u}$, be as in Lemma 2.1. Then there exists $\alpha=\alpha(p) \in(0,1)$ such that $\tilde{u}$ has a Hölder $\alpha$ continuous representative in $B(w, r)$ (also denoted $\tilde{u}$ ). Moreover if $x, y \in B(w, r)$ then

$$
|\tilde{u}(x)-\tilde{u}(y)| \leq c(|x-y| / r)^{\alpha} \max _{B(w, 2 r)} \tilde{u} .
$$

Lemma 2.3. Let $p, \Omega, w, r, \tilde{u}$, be as in Lemma 2.1 and let $\tilde{\mu}$ be the measure associated with $\tilde{u}$ as in (1.2). Then there exists $c$ such that

$$
c^{-1} r^{p-2} \tilde{\mu}[B(w, r / 2)] \leq \max _{B(w, r)} \tilde{u}^{p-1} \leq c r^{p-2} \tilde{\mu}[B(w, 2 r)] .
$$

Using Lemma 2.3 we prove,

Lemma 2.4. Fix $p, 1<p<\infty$, and let $\hat{u}$ be the positive $p$ harmonic function in Theorem 1. Also, let $u$ be the $p$ capacitary function for $D=\Omega \backslash \bar{B}\left(z_{0}, d\left(z_{0}, \partial \Omega\right) / 2\right)$, defined below Corollary 1 , and let $\mu, \hat{\mu}$, be the measures corresponding to $u, \hat{u}$, respectively. Then $\mu, \hat{\mu}$ are mutually absolutely continuous. In particular, Theorem 1 is valid for $\hat{\mu}$ if and only if it is valid for $\mu$.

Proof: We note that if $\nu \not \equiv 0$ is a finite Borel measure on $\mathbb{C}$ with compact support, then

$$
\nu(\mathbb{C} \backslash \Gamma)=0 \text { where } \Gamma=\left\{z \in \operatorname{supp} \nu: \liminf _{t \rightarrow 0} \frac{\nu(B(z, 100 t))}{\nu(B(z, t))} \leq 10^{9}\right\}
$$

Indeed otherwise, there exists a Borel set $\Lambda \subset \mathbb{C}$ with $\nu(\Lambda)>0$ and the property that if $z \in \Lambda$, then there exists $t_{0}(z)>0$ for which

$$
\nu(B(z, t)) \leq 10^{-8} \nu(B(z, 100 t)) \text { for } 0<t<t_{0}(z) .
$$

Iterating (2.6) it follows that

$$
\lim _{t \rightarrow 0} \frac{\nu(B(z, t))}{t^{3}}=0 \text { whenever } z \in \Lambda
$$

Since $H^{3}(\mathbb{C})=0$, we deduce from $(2.7)$ that $\nu(\Lambda)=0$, which is a contradiction. Thus $(2.5)$ is true.

Now suppose that $\mu, \hat{\mu}$ are as in Lemma 2.4. Let $N_{1}$ be a neighborhood of $\partial \Omega$ with

$$
\partial \Omega \subset N_{1} \subset \bar{N}_{1} \subset N .
$$


Then from compactness and continuity of $\hat{u}, u$, there exists $\hat{M}<\infty$ such that

$$
u \leq \hat{M} \hat{u} \leq \hat{M}^{2} u
$$

on $\Omega \cap \partial N_{1}$. From (2.8) and the boundary maximum principle for $p$ harmonic functions we conclude that (2.8) holds in $\Omega \cap N_{1}$. In view of (2.8) and Lemma 2.3 we see there exists $\hat{r}>0$, and a constant $b<\infty$, such that

$$
\mu(B(w, s)) \leq b \hat{\mu}(B(w, 2 s)) \leq b^{2} \mu(B(w, 4 s))
$$

whenever $w \in \partial \Omega$ and $0<s \leq \hat{r}$. We also note from Lemma 2.3 that supp $\mu=\operatorname{supp} \hat{\mu}=\partial \Omega$.

The proof of Lemma 2.4 is by contradiction. Let $E \subset \partial \Omega$ be a Borel set with $\hat{\mu}(E)=0$. If $\mu(E)>0$, then from properties of Borel measures, and with $\Gamma$ as in $(2.5)$ with $\nu=\mu$, we see there exists a compact set $K$ with

$$
K \subset E \cap \Gamma \text { and } \mu(K)>0 .
$$

Given $\epsilon>0$ there also exists an open set $O$ with

$$
E \subset O \text { and } \hat{\mu}(O)<\epsilon \text {. }
$$

Moreover, we may suppose for each $z \in K$ that there is a $\rho=\rho(z)$ with $0<\rho(z)<\hat{r} / 1000$, $\bar{B}(z, 100 \rho(z)) \subset O$, and

$$
\mu(B(z, 100 \rho)) \leq 10^{10} \mu(B(z, \rho)) .
$$

Applying Vitali's covering theorem we then get $\left\{B\left(z_{i}, r_{i}\right)\right\}$ with $z_{i} \in \partial \Omega, 0<100 r_{i}<\hat{r}$ and the property that

(a) (2.12) holds with $\rho=r_{i}$ for each $i$,

(b) $K \subset \bigcup_{i} B\left(z_{i}, 100 r_{i}\right) \subset O$,

(c) $B\left(z_{i}, 10 r_{i}\right) \cap B\left(z_{j}, 10 r_{j}\right)=\emptyset$ when $i \neq j$.

Using (2.9) and (2.11) - (2.13), it follows that

$$
\begin{aligned}
\mu(K) & \leq \mu\left[\cup_{i} B\left(z_{i}, 100 r_{i}\right)\right] \leq \sum_{i} \mu\left[B\left(z_{i}, 100 r_{i}\right)\right] \leq 10^{10} \sum_{i} \mu\left[B\left(z_{i}, r_{i}\right)\right] \\
& \leq 10^{10} b \sum_{i} \hat{\mu}\left[B\left(z_{i}, 10 r_{i}\right)\right] \leq 10^{10} b \hat{\mu}(O) \leq 10^{10} b \epsilon
\end{aligned}
$$

Since $\epsilon$ is arbitrary we conclude that $\mu(K)=0$, which contradicts (2.10). Thus $\mu$ is absolutely continuous with respect to $\hat{\mu}$. Interchanging the roles of $\mu, \hat{\mu}$ we also get that $\hat{\mu}$ is absolutely continuous with respect to $\mu$. Thus Lemma 2.4 is true. 


\section{Proof of Theorem 1 (assuming Theorem 2).}

From Lemma 2.4 we see that it suffices to prove Theorem 1 with $\hat{u}, \hat{\mu}$, replaced by $u, \mu$. In this section we prove Theorem 1 for $u$ under the assumption that Theorem 2 is correct. Given Theorem 2 we can follow closely the argument in [BL05] from (6.9) on. However, our argument is necessarily somewhat more complicated, as in [BL05] we used the fact that $\mu$ was a doubling measure, which is not necessarily true when $\Omega$ is simply connected. We claim that it suffices to prove Theorem 1 when

$$
z_{0}=0 \text { and } d\left(z_{0}, \partial \Omega\right)=2 .
$$

Indeed, let $\tau=d\left(z_{0}, \partial \Omega\right) / 2$ and put $T(z)=z_{0}+\tau z$. If $u^{\prime}(z)=u(T(z))$ for $z \in D$, then since the $p$ Laplacian is invariant under translations, rotations, dilations, it follows that $u^{\prime}$ is $p$ harmonic in $T^{-1}(D)$. Let $\mu^{\prime}$ be the measure corresponding to $u^{\prime}$. Then from (1.2) it follows easily that

$$
\mu^{\prime}(E)=\tau^{p-2} \mu(T(E)) \text { whenever } E \subset \mathbb{R}^{n} \text { is a Borel set. }
$$

This equality clearly implies that H-dim $\mu^{\prime}=\mathrm{H}$-dim $\mu$. Thus we may assume that (3.1) holds. Then $B(0,2) \subset \Omega$ and $D=\Omega \backslash \bar{B}(0,1)$.

Using Theorem 2 we have, for some $c=c(p) \geq 1$, that

$$
c^{-1} \frac{u(z)}{d(z, \partial \Omega)} \leq|\nabla u(z)| \leq c \frac{u(z)}{d(z, \partial \Omega)} \text { whenever } z \in D
$$

Next set

$$
v(x)=\left\{\begin{array}{l}
\max (\log |\nabla u(x)|, 0) \text { when } 1<p<2 \\
\max (-\log |\nabla u(x)|, 0) \text { when } p>2
\end{array}\right.
$$

Then in [BL05] it is shown that

$$
\int_{\{x: u(x)=t\}}|\nabla u|^{p-1} \exp \left[\frac{w^{2}}{2 c_{+} \log (1 / t)}\right] d H^{1} x \leq 2 c_{+}
$$

for some $c^{+} \geq 1$. In [BL05], $c^{+}$depends on $k, p$, but only because the constant in (3.2) depends on $k, p$. So, given Theorem $2, c^{+}=c^{+}(p)$ in (3.3). Next let

$$
\begin{gathered}
\xi(t)=2 \sqrt{c_{+} \log (1 / t) \log \log (1 / t)} \text { for } 0<t<10^{-6}, \\
F(t)=\{x: u(x)=t \text { and } v(x) \geq \xi(t)\}
\end{gathered}
$$

Then from (3.3) and weak type estimates we deduce

$$
\int_{F(t)}|\nabla u|^{p-1} d H^{1} x \leq 2 c_{+}[\log (1 / t)]^{-2} .
$$

Next for $A$ fixed with $|A|$ large, we define $\lambda$ as in Theorem 1 . Let $a=\frac{|A|}{2 \sqrt{c^{+}}}$and note that

$$
\lambda(r)=\left\{\begin{array}{l}
r e^{a \xi(r)} \text { when } 1<p<2 \\
r e^{-a \xi(r)} \text { when } p>2
\end{array}\right.
$$


To prove Theorem 1 when either $1<p<2$ or $p>2$, we intially allow $a$ to vary but will later fix it as a constant depending only on $p$, satisfying several conditions. Fix $p, 1<p<2$, and let $K \subset \partial \Omega$ be a Borel set with $H^{\lambda}(K)=0$. Let $K_{1}$ be the subset of all $z \in K$ with

$$
\limsup _{r \rightarrow 0} \frac{\mu(B(z, r))}{\lambda(r)}<\infty .
$$

Then from the definition of $\lambda$ and a covering argument (see [Mat95, sec 6.9]), it is easily shown that $\mu\left(K_{1}\right)=0$. Thus to prove $\mu(K)=0$, it suffices to show $\mu(E)=0$ when $E$ is Borel and is equal $\mu$ almost everywhere to the set of all points in $\partial \Omega$ for which

$$
\limsup _{r \rightarrow 0} \frac{\mu(B(z, r))}{\lambda(r)}=\infty .
$$

Let $G$ be the set of all $z$ where (3.6) holds. Given $0<r_{0}<10^{-100}$, we first show for each $z \in G$ that there exists $s=s(z), 0<s / 100<r_{0}$, such that

$$
\mu(B(z, 100 s)) \leq 10^{9} \mu(B(z, s)) \text { and } \lambda(100 s) \leq \mu(B(z, s)) .
$$

In fact let $s \in\left(0, r_{0}\right)$ be the first point starting from $r_{0}$ where

$$
\frac{\mu(B(z, s))}{\lambda(s)} \geq 10^{20} \min \left\{\frac{\mu\left(B\left(z, r_{0}\right)\right)}{\lambda\left(r_{0}\right)}, 1\right\} .
$$

From (3.6) we see that $s$ exists. Using $\lambda(100 r) \leq 200 \lambda(r), 0<r<r_{0} / 100$, it is also easily checked that (3.7) holds. From (3.7) and Vitali again, we get $\left\{B\left(z_{i}, r_{i}\right)\right\}$ with $z_{i} \in G, 0<$ $100 r_{i}<r_{0}$, and the property that

(a) (3.7) holds with $z=z_{i}, s=r_{i}$, for each $i$,

(b) $\quad G \subset \bigcup_{i} B\left(z_{i}, 100 r_{i}\right)$

(c) $B\left(z_{i}, 10 r_{i}\right) \cap B\left(z_{j}, 10 r_{j}\right)=\emptyset$ when $i \neq j$.

Let $t_{m}=2^{-m}$ for $m=1,2, \ldots$ Given $i$, we claim there exists $w_{i} \in B\left(z_{i}, 5 r_{i}\right)$ and $m=m(i)$ with

$(\alpha) \quad u\left(w_{i}\right)=t_{m}$ and $d\left(w_{i}, \partial \Omega\right) \approx r_{i}$

( $\beta) \quad \mu\left[B\left(z_{i}, 10 r_{i}\right)\right] / r_{i} \approx\left[u\left(w_{i}\right) / d\left(w_{i}, \partial \Omega\right)\right]^{p-1} \approx|\nabla u(w)|^{p-1}$

whenever $w \in B\left(w_{i}, d\left(w_{i}, \partial \Omega\right) / 2\right)$.

In (3.9) all proportionality constants depend only on $p$. To prove (3.9) choose $\zeta_{i} \in \partial B\left(z_{i}, 2 r_{i}\right)$ with $u\left(\zeta_{i}\right)=\max _{\bar{B}\left(z_{i}, 2 r_{i}\right)} u$. Then $d\left(\zeta_{i}, \partial \Omega\right) \approx r_{i}$, since otherwise, it would follow from Lemma 2.2 that $u\left(\zeta_{i}\right)$ is small in comparison to $\max _{\bar{B}\left(z_{i}, 5 r_{i}\right)} u$. However from (3.8) (a) and Lemma 2.3, these two maximums are proportional with constants depending only on $p$. Thus $d\left(\zeta_{i}, \partial \Omega\right) \approx r_{i}$. Using this fact, $(3.2),(3.8)(a)$, and Lemma 2.3, once again we get $(3.9)(\beta)$ with $w_{i}$ replaced by $\zeta_{i}$. If $t_{m} \leq u\left(\zeta_{i}\right)<t_{m-1}$ we let $w_{i}$ be the first point on the line segment connecting $\zeta_{i}$ to a point in 
$\partial \Omega \cap \partial B\left(\zeta_{i}, d\left(\zeta_{i}, \partial \Omega\right)\right)$ where $u=t_{m}$. From our construction, Harnack's inequality, and Lemma 2.2 we see that $(3.9)$ is true.

Using (3.8), (3.9), we deduce for $1<p<2$ that

$$
v(z)=\log |\nabla u(z)| \geq a \xi\left(100 r_{i}\right) / \tilde{c} \text { on } B\left(w_{i}, d\left(w_{i}, \partial \Omega\right) / 2\right)
$$

where $a$ is as in (3.5). Next we note that

$$
H^{1}\left[B\left(w_{i}, d\left(w_{i}, \partial \Omega\right) / 2\right) \cap\left\{z: u(z)=t_{m}\right\}\right] \geq d\left(w_{i}, \partial \Omega\right) / 2
$$

as we see from the maximum principle for $p$ harmonic functions, a connectivity argument and basic geometry. Also, we can use (3.8) (a) to estimate $t_{m}$ below in terms of $r_{i}$ and Lemma 2.2 to estimate $t_{m}$ above in terms of $r_{i}$. Doing this we find for some $\beta=\beta(p), 0<\beta<1, \bar{c}=\bar{c}(p)$, that

$$
r_{i} \leq \bar{c} t_{m}^{\beta} \leq \bar{c}^{2} r_{i}^{\beta^{2}} .
$$

Using (3.8)-(3.12) we conclude, for $a$ large enough, that

$$
\mu\left[B\left(z_{i}, 10 r_{i}\right)\right] \leq c \int_{F\left(t_{m}\right) \cap B\left(z_{i}, 10 r_{i}\right)}|\nabla u|^{p-1} d H^{1} .
$$

Using (3.8), (3.12), (3.13), and (3.4) it follows for $c$ large enough that

$$
\begin{aligned}
\mu(G) & \leq \mu\left(\bigcup_{i} B\left(z_{i}, 100 r_{i}\right)\right) \leq 10^{9} \sum_{i} \mu\left[B\left(z_{i}, 10 r_{i}\right)\right] \\
& \leq c \sum_{m=m_{0}}^{\infty} \int_{F\left(t_{m}\right)}|\nabla u|^{p-1} d H^{1} x \leq c^{2} \sum_{m=m_{0}}^{\infty} m^{-2} \leq c^{3} m_{0}^{-1}
\end{aligned}
$$

where $2^{-m_{0} \beta}=\bar{c} r_{0}^{\beta^{2}}$. Since $r_{0}$ can be arbitrarily small we see from (3.14) that $\mu(G)=0$. This equality and the remark above (3.6) yield $\mu(K)=0$. Hence $\mu$ is absolutely continuous with respect to $H^{\lambda}$ and Theorem 1 is true for $1<p<2$.

Finally to prove Theorem 1 for $p>2$, we show there exists a Borel set $\hat{K} \subset \partial \Omega$ such that

$$
\mu(\hat{K})=\mu(\partial \Omega) \text { and } \hat{K} \text { has } \sigma \text { finite } H^{\lambda} \text { measure. }
$$

In fact let $\hat{K}$ be the set of all $z \in \partial \Omega$ with

$$
\limsup _{r \rightarrow 0} \frac{\mu(B(z, r))}{\lambda(r)}>0 .
$$

Let $\hat{K}_{n}$ be the subset of $\hat{K}$ where the above lim sup is greater than $1 / n$. Then from the definition of $\lambda$ and a Vitali covering type argument (see [Mat95, ch 2]) it follows easily that

$$
H^{\lambda}\left(\hat{K}_{n}\right) \leq 100 n \mu\left(\hat{K}_{n}\right) .
$$

Since $\hat{K}=\cup_{n} \hat{K}_{n}$ we conclude that $\hat{K}$ is $\sigma$ finite with respect to $H^{\lambda}$ measure. Thus to prove (3.15) it suffices to show $\mu(\hat{G})=0$ where $\hat{G}$ is equal to the set of all points in $\partial \Omega$ for which

$$
\lim _{r \rightarrow 0} \frac{\mu(B(z, r))}{\lambda(r)}=0 .
$$


Given $0<r_{0}<10^{-100}$ we argue as in the proof of $(2.5)$ to deduce for each $z \in \hat{G}$ the existence of $s=s(z), 0<s / 100<r_{0}$, such that

$$
\mu(B(z, 100 s)) \leq 10^{9} \mu(B(z, s)) \text { and } \lambda(s) \geq \mu(B(z, 100 s)) .
$$

Using (3.18) and once again applying Vitali's covering lemma we get $\left\{B\left(z_{i}, r_{i}\right)\right\}$ with $z_{i} \in$ $\hat{G}, 0<100 r_{i}<r_{0}$, and the property that

$$
\begin{aligned}
& \text { (a) }(3.18) \text { holds with } z=z_{i}, s=r_{i} \text { for each } i, \\
& \text { (b) } \hat{G} \subset \bigcup_{i} B\left(z_{i}, 100 r_{i}\right), \\
& \text { (c) } B\left(z_{i}, 10 r_{i}\right) \cap B\left(z_{j}, 10 r_{j}\right)=\emptyset \text { when } i \neq j
\end{aligned}
$$

Let $\Theta$ be the set of all indexes, $i$, for which $\mu\left(B\left(z_{i}, 100 r_{i}\right)\right) \geq r_{i}^{3}$ and let $\Theta_{1}$ be the indexes for which this inequality is false. Arguing as in (3.14) we obtain

$$
\mu(\hat{G}) \leq \bigcup_{i \in \Theta} \mu\left(B\left(z_{i}, 100 r_{i}\right)\right)+\sum_{i \in \Theta_{1}} r_{i}^{3} \leq 10^{9} \bigcup_{i \in \Theta} \mu\left(B\left(z_{i}, 10 r_{i}\right)\right)+100 r_{0}\left(H^{2}(\Omega)+1\right) .
$$

If $i \in \Theta$, we can repeat the argument after (3.8) to get (3.9). (3.9) and (3.8) (a) imply (3.10) for $w=-\log |\nabla u|$. Also since $i \in \Theta$ we can use (3.9) to estimate $t_{m}$ from below in terms of $r_{i}$ and once again use Lemma 2.2 to estimate $t_{m}$ from above in terms of $r_{i}$. Thus (3.12) also holds for some $\beta, \bar{c}$ depending only on $p$. (3.10) - (3.12) imply (3.13) for $a$ (as in (3.5)) suitably large. In view of $(3.20),(3.13)$, and (3.4) we have

$$
\begin{aligned}
\mu(\hat{G})-100 r_{0}\left(H^{2}(\Omega)+1\right) & \leq \mu\left(\bigcup_{i} B\left(z_{i}, 100 r_{i}\right)\right) \leq 10^{9} \sum_{i \in \Theta} \mu\left[B\left(z_{i}, 10 r_{i}\right)\right] \\
& \leq c \sum_{m=m_{0}}^{\infty} \int_{F\left(t_{m}\right)}|\nabla u|^{p-1} d H^{1} x \leq c^{2} \sum_{m=m_{0}}^{\infty} m^{-2} \leq c^{3} m_{0}^{-1}
\end{aligned}
$$

where $2^{-m_{0} \beta}=\bar{c} r_{0}^{\beta^{2}}$. Since $r_{0}$ can be arbitrarily small we conclude first from $(3.21)$ that $\mu(\hat{G})=$ 0 and thereupon that (3.15) is valid. Hence $\mu$ is concentrated on a set of $\sigma$ finite $H^{\lambda}$ measure when $p>2$. The proof of Theorem 1 is now complete given that Theorem 2 is true.

\section{Preliminary Reductions for Theorem 2.}

Let $u$ be the $p$ capacitary function for $D=\Omega \backslash B\left(z_{0}, d\left(z_{0}, \partial \Omega\right) / 2\right)$. We extend $u$ to $\mathbb{C}$ by putting $u \equiv 1$ on $\bar{B}\left(z_{0}, d\left(z_{0}, \partial \Omega\right) / 2\right)$ and $u \equiv 0$ in $\mathbb{C} \backslash \Omega$. We shall need some more basic properties of $u$. Again references for proofs can be found in [BL05].

Lemma 4.1. If $z=x+i y, i=\sqrt{-1}, x, y \in \mathbb{R}$, then $u_{z}=(1 / 2)\left(u_{x}-i u_{y}\right)$ is a quasi-regular mapping of $D$ and $\log |\nabla u|$ is a weak solution to a linear elliptic PDE in divergence form in $D$. Moreover, positive weak solutions to this $P D E$ in $B(\zeta, r) \subset D$ satisfy the Harnack inequality

$$
\max _{B(\zeta, r / 2)} h \leq \tilde{c} \min _{B(\zeta, r / 2)} h
$$


where $\tilde{c}$ depends only on $p$.

Lemma 4.2. $u$ is real-analytic in $D, \nabla u \neq 0$ in $D$, and $\nabla u$ has a Hölder continuous extension to a neighborhood of $\partial B\left(z_{0}, d\left(z_{0}, \partial \Omega\right) / 2\right)$. Moreover, there are constants $\beta, 0<\beta<1$, and $\hat{c} \geq 1$, depending only on $p$, such that

$$
|\nabla u(z)-\nabla u(w)| \leq \hat{c}\left(\frac{|z-w|}{d(z, \partial \Omega)}\right)^{\beta} \max _{B(z, d(z, \partial \Omega) / 2)}|\nabla u| \leq \hat{c}^{2}\left(\frac{|z-w|}{d(z, \partial \Omega)}\right)^{\beta} \frac{u(z)}{d(z, \partial \Omega)}
$$

whenever $w \in D \cap B(z, d(z, \partial \Omega) / 2)$. Finally

$$
\hat{c}|\nabla u(w)| \geq \frac{u(w)}{d(w, \partial \Omega)} \text { for } w \in D \cap B\left(z_{0}, 3 d\left(z_{0}, \partial \Omega\right) / 4\right) .
$$

Using Lemma 4.2 we see that Theorem 2 is true when $z \in D \cap B\left(z_{0}, 3 d\left(z_{0}, \partial \Omega\right) / 4\right)$. Thus it is enough to prove Theorem 2 with $z=z_{1}$ for

$$
z_{1} \in D \backslash B\left(z_{0}, 3 d\left(z_{0}, \partial \Omega\right) / 4\right) .
$$

Recall the definition of the hyperbolic distance $\rho_{\Omega}$ for a simply connected domain $\Omega$ (see [GM05]). Then $\rho_{\Omega}\left(z_{1}, z_{2}\right), z_{1}, z_{2} \in \Omega$, is comparable to the quasi-hyperbolic distance

$$
Q_{\Omega}\left(z_{1}, z_{2}\right):=\inf \int_{\gamma} \frac{|d z|}{d(z, \partial \Omega)}
$$

where the infimum is taken over all the paths $\gamma \subset \Omega$ connecting $z_{1}$ to $z_{2}$. More specifically,

$$
\rho_{\Omega} \leq Q_{\Omega} \leq 4 \rho_{\Omega}
$$

as follows from the Koebe estimates

$$
\frac{1}{4}\left|f^{\prime}(z)\right|\left(1-|z|^{2}\right) \leq d(f(z), \partial \Omega) \leq\left|f^{\prime}(z)\right|\left(1-|z|^{2}\right), z \in B(0,1),
$$

whenever $f: B(0,1) \rightarrow \Omega$ is a conformal map, (see Theorem I.4.3 in [GM05]). In the following we will often use the following distortion estimate, which also follows from Koebe's Theorem, (see (I.4.17) in [GM05]), for conformal maps $f: B(0,1) \rightarrow \mathbb{C}$. For $z_{1}, z_{2} \in D$,

$$
\rho_{\Omega}\left(z_{1}, z_{2}\right) \leq A_{1} \Longrightarrow\left|f^{\prime}\left(f^{-1}\left(z_{2}\right)\right)\right| \leq A_{2}\left|f^{\prime}\left(f^{-1}\left(z_{1}\right)\right)\right|
$$

for some constant $A_{2}$ depending only on $A_{1}$. Note also that (4.6) implies that $d\left(z_{2}, \partial \Omega\right) \leq$ $A_{3} d\left(z_{1}, \partial \Omega\right)$ for some constant $A_{3}$ depending only on $A_{2}$. The same holds if $f$ is a conformal mapping of the upper half-plane $\mathbb{H}$. Our main lemma in the proof of Theorem 2 is the following.

Lemma 4.7. There is a constant $C$, depending only on $p$, such that if $z_{1}$ is as in (4.3) then there exists $z^{\star} \in \Omega$ with $u\left(z^{\star}\right)=u\left(z_{1}\right) / 2$ and $\rho_{\Omega}\left(z_{1}, z^{\star}\right) \leq C$.

Assuming for the moment that Lemma 4.7 is proved we get Theorem 2 from the following argument. Let $\Gamma$ be the hyperbolic geodesic connecting $z_{1}$ to $z^{*}$. If $\Gamma \cap B\left(z_{0}, 5 d\left(z_{0}, \partial \Omega\right) / 8\right)=\emptyset$, 
we put $\gamma=\Gamma$. Otherwise, $\gamma=\gamma_{1}+\gamma_{2}+\gamma_{3}$ where $\gamma_{1}$ is the subarc of $\Gamma$ joining $z_{1}$ to the first point, $P_{1}$, where $\Gamma$ intersects $\partial B\left(z_{0}, 5 d\left(z_{0}, \partial \Omega\right) / 8\right) ; \gamma_{2}$ is the short arc of $\partial B\left(z_{0}, 5 d\left(z_{0}, \partial \Omega\right) / 8\right)$ joining $P$ to the last point, $P_{2}$, where $\gamma$ intersects $\partial B\left(z_{0}, 5 d\left(z_{0}, \partial \Omega\right) / 8\right)$; and finally $\gamma_{3}$ joins $P_{2}$ to $z^{*}$. Using (4.3)-(4.6), one sees that

$$
H^{1}(\gamma) \leq c d\left(z_{1}, \partial \Omega\right) \text { and } d(\gamma, \partial \Omega) \geq c^{-1} d\left(z_{1}, \partial \Omega\right)
$$

where $c=c(p)$. Thus

$$
\frac{1}{2} u\left(z_{1}\right) \leq u\left(z_{1}\right)-u\left(z^{\star}\right) \leq \int_{\gamma}|\nabla u(z)||d z| \leq c H^{1}(\gamma) \max _{\gamma}|\nabla u| \leq C d\left(z_{1}, \partial \Omega\right) \max _{\gamma}|\nabla u| .
$$

So for some $\zeta \in \gamma$,

$$
c^{\star}|\nabla u(\zeta)| \geq \frac{u\left(z_{1}\right)}{d\left(z_{1}, \partial \Omega\right)}
$$

where $c^{\star} \geq 1$ depends only on $p$. Also from (4.8) we deduce the existence of balls $\left\{B\left(w_{j}, r_{j}\right\}_{j=1}^{N}\right.$, with $w_{j} \in \gamma$ and

(a) $B\left(w_{j}, r_{j} / 4\right) \cap B\left(w_{j+1}, r_{j+1} / 4\right) \neq \emptyset$ for $1 \leq j \leq N-1$

(b) $\quad r_{j} \approx d\left(B\left(w_{j}, r_{j}\right), \partial \Omega\right) \approx d\left(z_{1}, \partial \Omega\right)$,

(c) $\quad \gamma \subset \bigcup_{j} B\left(w_{j}, r_{j} / 4\right)$,

where $N$ and proportionality constants depend only on $p$. Observe from (4.10) and Harnack's inequality applied to $u$ (see Lemma 2.1) that $u(z) \approx u\left(z_{1}\right)$ when $z \in \cup_{j} B\left(w_{j}, r_{j}\right)$. In view of Lemma 4.2, (4.10), it follows for some $c=c(p)$ that

$$
|\nabla u(z)| \leq c u\left(z_{1}\right) / d\left(z_{1}, \partial \Omega\right) \text { when } z \in \bigcup_{j} B\left(w_{j}, r_{j} / 2\right) .
$$

From (4.11) we see that if $c=c(p) \geq 1$ is large enough and

$$
h(z)=: \log \left(\frac{c u\left(z_{1}\right)}{d\left(z_{1}, \partial \Omega\right)|\nabla u(z)|}\right) \text { for } z \in \bigcup_{j} B\left(w_{i}, r_{i} / 2\right)
$$

then $h>0$ in $\cup_{i} B\left(w_{i}, r_{i} / 2\right)$. Choose $i, 1 \leq i \leq N$, so that $\zeta \in B\left(w_{i}, r_{i} / 4\right)$. Using (4.9) we have $h(\zeta) \leq c$. Applying the Harnack inequality in Lemma 4.1 to $h$ in $B\left(w_{i}, r_{i} / 2\right)$ we get

$$
c|\nabla u| \geq u\left(z_{1}\right) / d\left(z_{1}, \partial \Omega\right) \text { in } B\left(w_{i}, r_{i} / 4\right) .
$$

From (4.10) we see that the argument leading to (4.12) can be repeated in a chain of balls connecting $\zeta$ to $z_{1}$. Doing this and using $N=N(p)$, we get Theorem 2 .

In the proof of Lemma 4.7 we may assume without loss of generality that $\partial \Omega$ is an analytic Jordan curve, as the constant in this lemma will depend only on $p$. Indeed, we can approximate $\Omega$ by an increasing sequence of analytic Jordan domains $\Omega_{n} \subset \Omega$, and apply Lemma 4.7 to $u_{n}$ the $p$ capacitary function for $D_{n}=\Omega_{n} \backslash B\left(z_{0}, d\left(z_{0}, \partial \Omega\right) / 2\right)$. Doing this and letting $n \rightarrow \infty$, we get Lemma 4.7 for $u$, since by Lemmas $2.2,4.2$, there are subsequences of $u_{n}, \nabla u_{n}$, converging to $u, \nabla u$, respectively, uniformly on compact subsets of $\Omega$. 


\subsection{Outline of the proof of Lemma 4.7.}

To prove Lemma 4.7 It will be useful to transfer the problem to the upper half-plane $\mathbb{H}$ via the Riemann map $f: \mathbb{H} \rightarrow \Omega$ such that $f(i)=z_{0}$ and $f(a)=z_{1}$ where $a=i s$ for some $0<s<1$. We note that $f$ has a continuous extension to $\overline{\mathbb{H}}$, since $\partial \Omega$ is a Jordan curve. We also let $U=u \circ f$, and note that $U$ satisfies a maximum principle and Harnack's inequality. Consider the box

$$
Q(a)=\{z=x+i y:|x| \leq s, 0<y<s\} .
$$

We will show that $Q(a)$ can be shifted to a nearby box $\tilde{Q}(a)$ whose boundary in $\mathbb{H}$ we call $\xi$. It consists of the horizontal segment from $x_{1}+i s$ to $x_{2}+i s$, and the vertical segments connecting $x_{l}+i s$ to $x_{l}$ for $l=1,2 . x_{1}, x_{2}$, are chosen to satisfy $-s<x_{1}<-s / 2, s / 2<x_{2}<s$. Let $f\left(x_{j}\right)=w_{j}, j=1,2 . \tilde{Q}(a)$ will be constructed to have several nice properties. In particular, we will prove that $U \leq A U(a)$, on $\xi$, and hence, by the maximum principle, $U \leq A U(a)$ on $\tilde{Q}(a)$, for some constant $A$ depending only on $p$. In other words, if we let $\sigma:=f(\xi)$ and $\Omega_{1}:=f(\tilde{Q}(a))$, then we will prove that

$$
u \leq A u\left(z_{1}\right)
$$

on $\sigma$ and hence in $\Omega_{1}$. Moreover, we will prove that

$$
H^{1}(\sigma) \leq C_{1} d\left(z_{1}, \partial \Omega\right)
$$

for some absolute constant $C_{1}$ depending only on $p$. Furthermore,we will establish the existence of $w_{0}=f\left(x_{0}\right)$, for some $\left|x_{0}\right|<s / 4$, such that $\left|w_{0}-z_{1}\right| \leq C_{2} d\left(z_{1}, \partial \Omega\right)$ and such that

$$
d\left(w_{0}, \sigma\right) \geq d\left(z_{1}, \partial \Omega\right) / C_{2}
$$

where $C_{2}$ is an other absolute constant. In addition we will construct a Lipschitz curve $\tau$ : $[0,1) \rightarrow \Omega_{1}$ with $\tau(0)=z_{1}$ and $\tau(1)=w_{0}$, which satisfies the cigar condition

$$
\min \left\{H^{1}(\tau[0, t]), H^{1}(\tau[t, 1])\right\} \leq C_{3} d(\tau(t), \partial \Omega),
$$

for $0 \leq t \leq 1$ and some absolute constant $C_{3}$.

To briefly outline the construction of $\tau$ we note that we construct $\tau$ as the image under $f$ of a polygonal path

$$
\lambda=\sum_{k=1}^{\infty} \lambda_{k} \subset \tilde{Q}(a),
$$

starting at $a$ and tending to $x_{0}$ non-tangentially. The segment $\lambda_{k}, k=1,2, \ldots$, joins $a_{k-1}$ to $a_{k}$ and consists of a horizontal line segment followed by a downward pointing vertical segment. More precisely, fix $\delta, 0<\delta<10^{-1000}$ and put $\delta^{*}=e^{-c^{*} / \delta}, t_{0}=0, s_{0}=s, a_{0}=t_{0}+i s_{0}=a$. In our construction we initially allow $\delta$ to vary but shall fix $\delta$ in (5.3) to be a small positive absolute constant satisfying several conditions. Also, $c^{*} \geq 1$ is an absolute constant which will be defined in Lemma 4.26. Then $\lambda_{1}$ consists of the horizontal segment from $a_{0}$ to $t_{1}+i s_{0}$ followed by the vertical segment from $t_{1}+i s_{0}$ to $a_{1}=t_{1}+i \delta^{*} s_{0}$. Put $s_{1}=\delta^{*} s_{0}$. Inductively, if $a_{k-1}=t_{k-1}+i s_{k-1}$ has been defined, then $\lambda_{k}$ consists of the horizontal line segment joining $a_{k-1}$ to $t_{k}+i s_{k-1}$, followed by the vertical line segment connecting $t_{k}+i s_{k-1}$ to $a_{k}=t_{k}+i s_{k}$, where $s_{k}=\delta^{*} s_{k-1}$. Moreover the numbers $t_{k}, k=1,2, \ldots$, are chosen in such a way that

$$
\left|t_{k}-t_{k-1}\right| \leq s_{k-1} \text { and } \int_{0}^{s_{k}}\left|f^{\prime}\left(t_{k}+i \tau\right)\right| d \tau \leq \delta d\left(f\left(a_{k-1}\right), \partial \Omega\right) .
$$


Existence of $\left(t_{k}\right)$ will be shown in the paragraph after (5.2). Letting $\tau_{k}=f\left(\lambda_{k}\right)$ and $z_{k}=$ $f\left(a_{k-1}\right), k=1,2, \ldots$, we note that (4.17) and our construction imply

$$
d\left(z_{k+1}, \partial \Omega\right) \leq \delta d\left(z_{k}, \partial \Omega\right) .
$$

For $w \in \lambda_{k}$, (4.6) and our construction give a constant $\bar{c}$, depending only on $\delta$ and $p$, such that

$$
\bar{c}^{-1}\left|f^{\prime}\left(a_{k-1}\right)\right| \leq\left|f^{\prime}(w)\right| \leq \bar{c}\left|f^{\prime}\left(a_{k-1}\right)\right| \text { whenever } w \in \lambda_{k} .
$$

Consequently for some constant $c \geq 1$, depending only on $\delta$ and $p$,

$$
c d(w, \partial \Omega) \geq d\left(z_{k}, \partial \Omega\right) \text { when } w \in \tau_{k} \text { and } H^{1}\left(\tau_{k}\right) \leq c(\delta) d\left(z_{k}, \partial \Omega\right)
$$

for $k=1,2,3, \ldots$

Putting (4.18) and (4.19) together we see that if $w=\tau(t) \in \tau_{k}$, then for some $c_{+} \geq 1$, depending only on $\delta$ and $p$,

$$
\left|w-w_{0}\right| \leq H^{1}(\tau[t, 1]) \leq \sum_{j=k}^{\infty} H^{1}\left(\tau_{j}\right) \leq c_{+} d(w, \partial \Omega) \leq c_{+}^{2} \delta^{k-1} d\left(z_{1}, \partial \Omega\right) .
$$

Using this equality and (4.19) we conclude that $\tau$ satisfies the cigar condition in (4.16) with a constant depending only on $\delta, p$.

To show the existence of $z^{*}$ in Lemma 4.7, we suppose $\delta>0$ is now fixed as in (5.3) and suppose that $\lambda$ is parametrized by $[0,1]$ with $\lambda(0)=a$ and $\lambda(1)=x_{0}$. Let

$$
t^{\star}=\max \left\{t: U(\lambda(t))=\frac{1}{2} U(a)\right\}
$$

and put $a^{\star}=\lambda\left(t^{\star}\right)$ and $z^{\star}=f\left(a^{\star}\right)$. If $\rho=d\left(w_{0}, \sigma\right)$, Then from the definition of $\Omega_{1}$ above (4.13) we have

$$
B\left(w_{0}, \rho\right) \cap \Omega \subset \Omega_{1} .
$$

so from Lemma 2.2 applied to the restriction of $u$ to $\Omega_{1}$, (4.13), (4.15), and (4.16) we deduce for some $\tilde{c}=\tilde{c}(p)$ that

$$
\frac{1}{2} u\left(z_{1}\right)=u\left(z^{\star}\right) \leq \tilde{c}\left(\frac{d\left(z^{*}, \partial \Omega\right)}{\rho}\right)^{\alpha} \max _{B\left(w_{0}, \rho\right) \cap \Omega} u \leq \tilde{c}^{2} A\left(\frac{d\left(z^{*}, \partial \Omega\right)}{d\left(z_{1}, \partial \Omega\right)}\right)^{\alpha} u\left(z_{1}\right) .
$$

Thus

$$
d\left(z_{1}, \partial \Omega\right) \leq c d\left(z^{*}, \partial \Omega\right) \text { for some } c=c(p) .
$$

This inequality and (4.16) imply that there is a chain of $N=N(p)$ balls (as in (4.10)) connecting $z_{1}$ to $z^{*}$. Using this implication and once again (4.4) we conclude that $\rho_{\Omega}\left(z^{*}, z_{1}\right) \leq c$. This completes our outline of the proof of Lemma 4.7.

To finish the proof of Lemma 4.7 we show there exists $\delta>0, \sigma, \tau, c^{*},\left(\tau_{k}\right)_{1}^{\infty}$, for which (4.13) - (4.15) and (4.17), are true. 


\subsection{Several Lemmas.}

To set the stage for the proof of $(4.13)$ - (4.15) and (4.17) we shall need several lemmas. To this end define, for $b \in \mathbb{H}$, the interval $I(b):=[\operatorname{Re} b-\operatorname{Im} b, \operatorname{Re} b+\operatorname{Im} b]$.

Lemma 4.20. There is an absolute constant $\hat{C}$ such that if $f$ is univalent on $\mathbb{H}$ and $b \in \mathbb{H}$, then

$$
\iint_{\mathbb{H}} \frac{\left|f^{\prime}(w)\right|}{|f(w)-f(b)|} d A(w) \leq \hat{C} \operatorname{Im} b .
$$

Proof of Lemma 4.20: The proof is left as an exercise. Hints are provided in problem 21 on page 33 of [GM05], where the case for functions $g$ univalent on $B(0,1)$ with $\operatorname{Re} g \neq 0$ is discussed. The same arguments give the result for univalent functions $g$ on $B(0,1)$ with $g(0)=0$ and then Lemma 4.20 is obtained by applying the result to $g=f \circ M_{b}$ where $M_{b}(z)=i \operatorname{Im} b(1+z) /(1-z)+\operatorname{Re} b$.

Lemma 4.21. There is a set $E(b) \subset I(b)$ such that for $x \in E(b)$

$$
\int_{0}^{\operatorname{Im} b}\left|f^{\prime}(x+i y)\right| d y \leq C^{\star} d(f(b), \partial \Omega)
$$

for some absolute constant $C^{\star}$, and also

$$
H^{1}(E(b)) \geq\left(1-10^{-100}\right) H^{1}(I(b)) .
$$

Note that we could achieve Lemma 4.21 by invoking known results in the literature, such as the result in [BB99] related to previous theorems of Beurling and Pommerenke (see [P75], Section 10.3). For completeness we give an alternative proof of Lemma 4.21 based on Lemma 4.20 .

Proof of Lemma 4.21: Let $\ell$ be a large positive integer that will soon be fixed as an absolute number and let

$$
T=T(b)=\{z=x+i y:|x|<\operatorname{Im} b: y=\operatorname{Im} b\}
$$

be the top of the box $Q(b)$ defined at the beginning of subsection 4.1. Set

$$
K=K(b):=\left\{x \in I(b):|f(x+i t)-f(b)|>2^{\ell}\left|f^{\prime}(b)\right| \operatorname{Im} b \text { for some } 0<t<\operatorname{Im} b\right\} .
$$

Note that

$$
\left|\partial_{y} \log \right| f(z)-f(b)|| \leq \frac{\left|f^{\prime}(z)\right|}{|f(z)-f(b)|} .
$$

Also, for $z$ in the top $T$,

$$
|f(z)-f(b)| \leq 1000\left|f^{\prime}(b)\right| \operatorname{Im} b .
$$

Thus,

$$
\int_{0}^{\operatorname{Im} b} \frac{\left|f^{\prime}(x+i y)\right|}{|f(x+i y)-f(b)|} d y \geq \frac{\ell}{C}
$$


whenever $x \in K$. Integrating both sides over $K$ and using Lemma 4.20 we therefore find that

$$
H^{1}(K) \leq C \frac{\operatorname{Im} b}{\ell} .
$$

Next for we define a function $g(x)$ for $x \in I(b)$ as follows. If $x \in I(b) \backslash K$ we set

$$
g(x):=\int_{0}^{\operatorname{Im} b}\left|f^{\prime}(x+i y)\right| d y
$$

and if $x \in K$ then we set $g(x)=0$. From the definition of $K$ we see that

$$
g(x) \leq 2^{\ell}\left|f^{\prime}(b)\right| \operatorname{Im} b \int_{0}^{\operatorname{Im} b} \frac{\left|f^{\prime}(x+i y)\right|}{|f(x+i y)-f(b)|} d y
$$

whenever $x \in I(b)$. Using this inequality and Integrating over $I(b)$ we find that

$$
\int_{I(b)} g(x) d x \leq C 2^{\ell}\left|f^{\prime}(b)\right|(\operatorname{Im} b)^{2} \leq C^{2} 2^{\ell} d(f(b), \partial \Omega) \operatorname{Im} b .
$$

So from weak-type estimates, if

$$
K^{\prime}:=\left\{x \in I(b): g(x)>2^{2 \ell} d(f(b), \partial \Omega)\right\}
$$

then

$$
H^{1}\left(K^{\prime}\right) \leq C^{2} 2^{-\ell} \operatorname{Im} b,
$$

for some absolute constant $C$. Using (4.24) and (4.25) we can fix $\ell$ to be a large absolute number so that

$$
H^{1}\left(K \cup K^{\prime}\right)<10^{-100} \operatorname{Im} b .
$$

With $\ell$ thus fixed we put

$$
E(b):=I(b) \backslash\left(K \cup K^{\prime}\right)
$$

and conclude that Lemma 4.21 is valid.

Lemma 4.26. Let $b, C^{\star}$ be as in Lemma 4.21 and put $c^{*}=4\left(C^{\star}\right)^{2}$. Given $0<\delta<10^{-1000}$, let $\delta_{\star}=e^{-c^{*} / \delta}$. Then, whenever $x \in E(b)$ there is an interval $J=J(x)$ centered at $x$ with

$$
2 \delta_{\star} \operatorname{Im} b \leq H^{1}(J) \leq C \delta^{1 / 2} \operatorname{Im} b \leq \frac{\operatorname{Im} b}{10000}
$$

(for some absolute constant $C$ ) and a subset $F=F(x) \subset J$ with $H^{1}(F) \geq\left(1-10^{-100}\right) H^{1}(J)$ so that

$$
\int_{0}^{\delta_{\star} \operatorname{Im} b}\left|f^{\prime}(t+i y)\right| d y \leq \delta d(f(b), \partial \Omega) \quad \text { for every } t \in F .
$$


Proof of Lemma 4.26: Given $x \in E(b)$ put $b^{\prime}=x+i \operatorname{Im} b$ and let $y_{1}, 0<y_{1}<\operatorname{Im} b$, be such that

$$
d(f(x+i y), \partial \Omega)>\frac{\delta}{C^{\star}} d(f(b), \partial \Omega)
$$

for $y_{1}<y<\operatorname{Im} b$, but

$$
d(f(\hat{b}), \partial \Omega)=\frac{\delta}{C^{\star}} d(f(b), \partial \Omega)
$$

where $\hat{b}:=x+i y_{1}$. By (4.4), Lemma 4.21, and conformal invariance of hyperbolic distance,

$$
\log \frac{\operatorname{Im} b}{y_{1}} \leq \rho_{\mathbb{H}}\left(\hat{b}, b^{\prime}\right) \leq 4 Q_{\Omega}\left(f(\hat{b}), f\left(b^{\prime}\right)\right) \leq \frac{4 C^{\star}}{\delta d(f(b), \partial \Omega)} \int_{y_{1}}^{\operatorname{Im} b}\left|f^{\prime}(x+i y)\right| d y \leq \frac{4\left(C^{\star}\right)^{2}}{\delta},
$$

i.e., $y_{1} \geq \delta_{\star} \operatorname{Im} b$. Let $J=I(\hat{b})$ and $F=E(\hat{b})$. Then by Lemma $4.21, H^{1}(F) \geq(1-$ $\left.10^{-100}\right) H^{1}(J)$ and for $t \in E(\hat{b})$

$$
\int_{0}^{\delta_{\star} \operatorname{Im} b}\left|f^{\prime}(t+i y)\right| d y \leq C^{\star} d(f(\hat{b}), \partial \Omega)=\delta d(f(b), \partial \Omega) .
$$

Notice also that,

$$
H^{1}(J)=2 \operatorname{Im} \hat{b} \geq 2 \delta_{\star} \operatorname{Im} b .
$$

On the other hand, elementary distortion theorems for univalent functions (see for example [GM05, ch 1, section 4]) and the fact that $\hat{b} \in Q(b)$ yield for some absolute constant $C_{+} \geq 1$ that

$$
\delta / C^{*}=\frac{d(f(\hat{b}), \partial \Omega)}{d(f(b), \partial \Omega)} \geq\left(\frac{\operatorname{Im} \hat{b}}{C_{+} \operatorname{Im} b}\right)^{2} .
$$

Thus (4.27), (4.28) are valid and the proof of Lemma 4.26 is complete.

Lemma 4.29 Let $b, x \in E(b), J(x), F(x)$, be as in Lemma 4.26 and set $\hat{F}=\bigcup_{x \in E(b)} F(x)$. If $L \subset I(b)$ is an interval with $H^{1}(L) \geq \frac{\operatorname{Im} b}{100}$, then

$$
H^{1}(E(b) \cap \hat{F} \cap L) \geq \frac{\operatorname{Im} b}{1000} .
$$

Moreover, if $\left\{\tau_{1}, \tau_{2}, \ldots, \tau_{m}\right\}$ is a set of points in $I(b)$, then there exists $\tau_{m+1}$ in $E(b) \cap \hat{F} \cap L$ with

$$
\left|f\left(\tau_{m+1}\right)-f\left(\tau_{j}\right)\right| \geq \frac{d(f(b), \partial \Omega)}{10^{10} m^{2}} \text { whenever } 1 \leq j \leq m .
$$

Proof of Lemma 4.29: Given an interval $I$ let $\lambda I$ be the interval with the same center as $I$ and $\lambda$ times its length. Using Vitali, we see there exists $\left\{\hat{x}_{j}\right\} \subset E(b) \cap \frac{1}{2} L$ and $\left\{J\left(\hat{x}_{j}\right)\right\}$ as in Lemma 4.26 such that

$$
E(b) \cap \frac{1}{2} L \subset \bigcup_{j} 4 J\left(\hat{x}_{j}\right) \text { and the intervals }\left\{J\left(\hat{x}_{j}\right)\right\} \text { are pairwise disjoint. }
$$


Observe from (4.27) that $J\left(\hat{x}_{j}\right) \subset L$ for each $j$. From this fact and (4.27) we get

$$
\begin{aligned}
H^{1}(\hat{F} \cap L) \geq \sum_{j} H^{1}\left(\hat{F} \cap J\left(\hat{x}_{j}\right)\right) & \geq\left(1-10^{-100}\right) \sum_{j} H^{1}\left(J\left(\hat{x}_{j}\right)\right) \geq \frac{1-10^{-100}}{4} \sum_{j} H^{1}\left(4 J\left(\hat{x}_{j}\right)\right) \\
& \geq \frac{1-10^{-100}}{4} H^{1}\left(E(b) \cap \frac{1}{2} L\right) \geq \operatorname{Im} b / 900
\end{aligned}
$$

From (4.32) and (4.23) we conclude that (4.30) is valid. To prove (4.31) observe from (4.30) and the Poisson integral formula for $\mathbb{H}$ that

$$
\omega(E(b) \cap \hat{F} \cap L, b) \geq 10^{-4}
$$

where $\omega(\cdot, b)$ denotes harmonic measure on $\mathbb{H}$ relative to $b$. Let

$$
r=\sup _{x \in E(b) \cap \hat{F} \cap L} \min \left\{\left|f(x)-f\left(\tau_{j}\right)\right|, 1 \leq j \leq m\right\}
$$

Then

$$
f(E(b) \cap \hat{F} \cap L) \subset \bigcup_{j=1}^{m} \bar{B}\left(f\left(\tau_{j}\right), r\right) .
$$

Using this fact, (4.33), and invariance of harmonic measure under $f$, it follows that

$$
10^{-4} \leq \sum_{j=1}^{m} \tilde{\omega}\left(\bar{B}\left(f\left(\tau_{j}\right), r\right), f(b)\right)
$$

where $\tilde{\omega}(\cdot, f(b))$ denotes harmonic measure in $\Omega$ relative to $f(b)$. Finally we note from the Beurling projection theorem (see [GM05, ch 3, Corollary 9.3]) that for each $j$,

$$
\tilde{\omega}\left(\bar{B}\left(f\left(\tau_{j}\right), r\right), f(b)\right) \leq(4 / \pi)\left(\frac{r}{d(f(b), \partial \Omega)}\right)^{1 / 2} .
$$

Using this inequality in (4.34) we conclude that (4.31) is true. The proof of Lemma 4.29 is now complete.

\section{$5 \quad$ Proof of Theorem 2.}

\subsection{Proof of (4.14) and (4.15)}

Using Lemma 4.29 with $b=a=i s$, we deduce for given $\delta, 0<\delta<10^{-1000}$, the existence of $x_{1}, x_{2}, x_{3} \in E(a)$ with $-s<x_{1}<-s / 2,-\frac{1}{8} s<x_{3}<\frac{1}{8} s$, and $\frac{1}{2} s<x_{2}<s$, such that

$$
\begin{gathered}
\int_{0}^{\delta_{*} s}\left|f^{\prime}\left(x_{j}+i y\right)\right| d y \leq \delta d(f(a), \partial \Omega) \text { for } 1 \leq j \leq 3, \\
\min \left\{\left|f\left(x_{1}\right)-f\left(x_{3}\right)\right|,\left|f\left(x_{2}\right)-f\left(x_{3}\right)\right|\right\} \geq 10^{-11} d(f(a), \partial \Omega) .
\end{gathered}
$$


As earlier we let $\tilde{Q}(a)$ be the shifted box whose boundary in $\mathbb{H}, \xi$, consists of the horizontal line segment from $x_{1}+i s$ to $x_{2}+i s$, and the vertical line segments from $x_{j}$ to $x_{j}+i s$, for $j=1,2$. Also we put $\sigma=f(\xi)$ and note from $x_{j} \in E(a), j=1,2$, that (4.14) is valid. Moreover, we let $w_{i}=f\left(x_{i}\right)$ for $i \in\{1,2,3\}$. To construct $\tau$ as defined after (4.15), we put $t_{1}=x_{3}$ and continue as outlined above (4.17). In general if $a_{k-1}=t_{k-1}+i s_{k-1}$, we choose $t_{k} \in E\left(a_{k-1}\right)$ so that (4.17) holds with $s_{k}=\delta_{*} s_{k-1}$. This choice is possible thanks to Lemma 4.29. With $\lambda$ now defined note from the argument following (4.17) that $x_{0}=\lim _{t \rightarrow 1} \lambda(t)$ exists, $\left|x_{0}\right|<1 / 4$, and that $\tau=f(\lambda)$ satisfies the cigar condition in (4.16) for $t \in[0,1)$. If $w_{0}=\tau(1)$, then using (4.17), (4.18), we see that

$$
\left|w_{3}-w_{0}\right| \leq \hat{C} \delta d\left(z_{1}, \partial \Omega\right)
$$

for some absolute constant $\hat{C}$. From this inequality and (5.2), it follows that if

$$
\delta=\min \left(10^{-12} \hat{C}^{-1}, 10^{-1000}\right),
$$

then

$$
\min \left\{\left|w_{0}-w_{j}\right|, j=1,2\right\} \geq 10^{-12} d\left(z_{1}, \partial \Omega\right) .
$$

With $\delta$ now fixed, we see from (5.1) that the part of $\sigma$, say $\sigma_{1}$, corresponding to the vertical line segments from $x_{j}$ to $x_{j}+i \delta_{*} s, j=1,2$, satisfies

$$
d\left(\sigma_{1}, w_{0}\right) \geq 10^{-13} d\left(z_{1}, \partial \Omega\right)
$$

Using (4.4) we also get

$$
d\left(\sigma \backslash \sigma_{1}, \partial \Omega\right) \geq C^{-1} d\left(z_{1}, \partial \Omega\right)
$$

for some absolute constant $C$. Combining (5.5), (5.6), we obtain (4.15).

\subsection{Proof of $(4.13)$}

The proof of (4.13) is by contradiction. Suppose $u>A u\left(z_{1}\right)$ on $\sigma$. We shall obtain a contradiction if $A=A(p)$ is suitably large. Our argument is based on a recurrence type scheme often attributed to Carleson - Domar, see [C62], [D57], in the complex world, and to Caffarelli et. al., see [CFMS81], in the PDE world (see also [AS05] for references). Given the shifted box $\tilde{Q}(a)$ we let $b_{j, 1}=x_{j}+i \delta_{*} \operatorname{Im} a, j=1,2$, and note that $b_{j, 1}, j=1,2$, are points on the vertical sides of $\tilde{Q}(a)$. These points will spawn two new boxes $\tilde{Q}\left(b_{j, 1}\right), j=1,2$, which in turn will each spawn two more new boxes, and so on. Without loss of generality, we focus on $\tilde{Q}\left(b_{1,1}\right)$. This box is constructed in the same way as $\tilde{Q}(a)$ and we also construct, using Lemma 4.29 once again, a polygonal path $\lambda_{1,1}$ from $b_{1,1}$ to some point $x_{1,1} \in I\left(b_{1,1}\right)$, so that $\lambda_{1,1}$ is defined relative to $b_{1,1}$ in the same way that $\lambda$ was defined relative to $a$. There is only one caveat. Namely, the path $\lambda_{1,1}$ is required to be contained in the half-plane $\left\{\operatorname{Re} z<\operatorname{Re} b_{1,1}\right\}$, i.e., to stay entirely to the left of $b_{1,1}$. This extra caveat is easily achieved in view of Lemma 4.29. $\lambda_{2,1}$ with endpoints, $b_{2,1}, x_{2,1}$ is constructed similarly, to lie in $\left\{\operatorname{Re} z>\operatorname{Re} b_{2,1}\right\}$ (see Picture 1).

Next, using the Harnack inequality we see that there exists $\Lambda$ such that

$$
u(f(z)) \leq \Lambda u(f(a)) \text { whenever } z=x+i y \in \xi, y \geq \delta_{*} \operatorname{Im} a .
$$




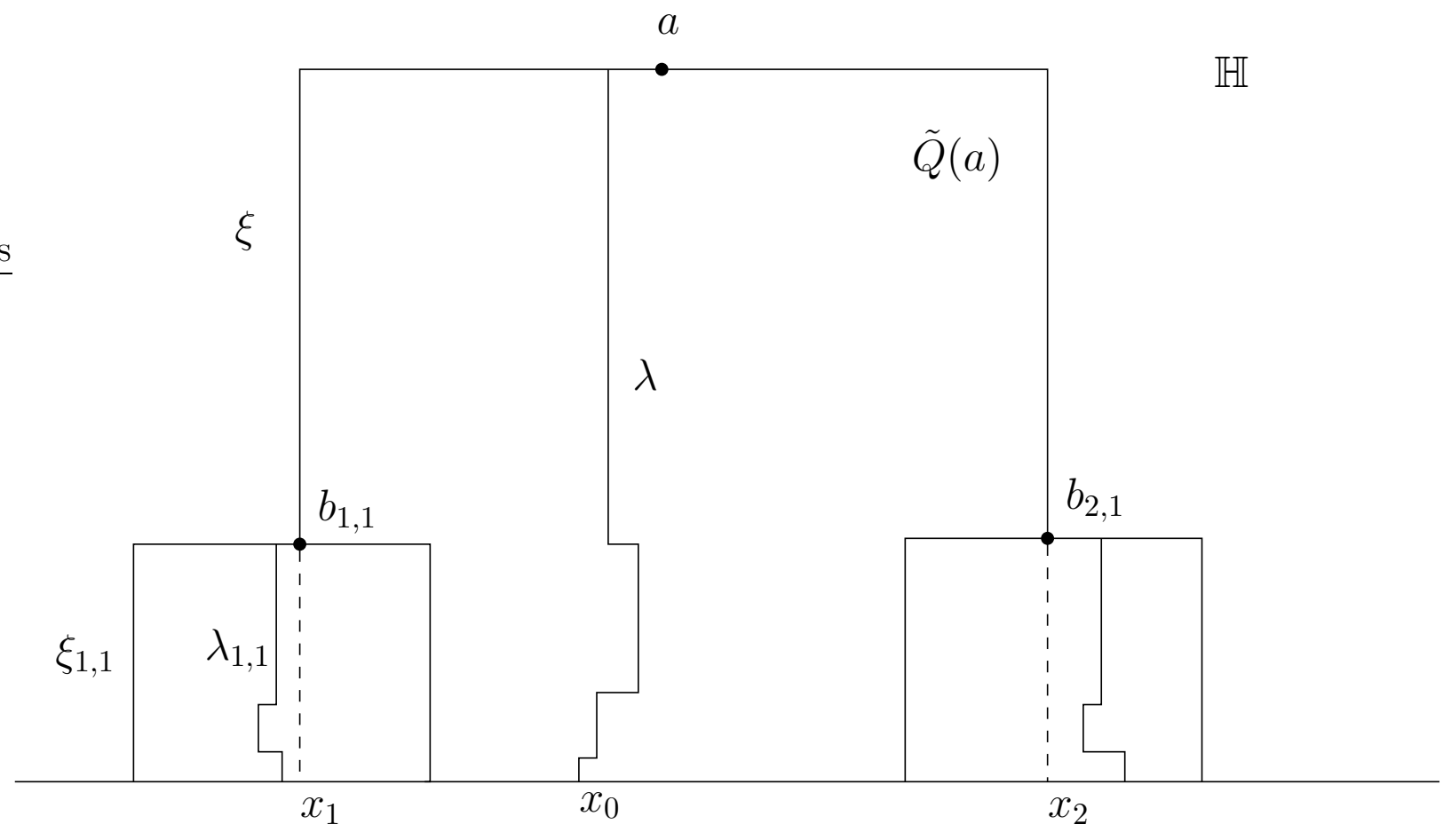

Figure 1: Domar-type recursion construction

In particular, from Harnack's inequality for $u$ and the fact that $\delta$ is now fixed in (5.3), it is clear that $\Lambda$ in (5.7) can be chosen to depend only on $p$, and hence can also be used in further iterations.

By (5.7), the fact that $A>\Lambda$ and the maximum principle, we see that there exists a point $z \in \lambda_{1,1} \cup \lambda_{2,1}$ such that $U(z)>A U(a)$. This is the reason why the paths $\lambda_{j, 1}$ are constructed outside the original box $\tilde{Q}(a)$. First suppose $z \in \lambda_{1,1}$. The larger the constant $A$, the closer $z$ will be to $\mathbb{R}$. More precisely, if $A>\Lambda^{k}$ then $\operatorname{Im} z \leq \delta_{\star}^{k} \operatorname{Im} a$, as we see from (5.7) and inequalities analogous to (4.17)-(4.19). Arguing as in the display below (4.19), we find that

$$
\left|f(z)-f\left(x_{1,1}\right)\right| \leq C \delta^{k-1} d\left(f\left(b_{1,1}\right), \partial \Omega\right) .
$$

The argument now is similar to the argument showing the existence of $z^{*}$ at the end of subsection 4.1. Let $\xi_{1,1}$ be the boundary of $\tilde{Q}\left(b_{1,1}\right)$ which is in $\mathbb{H}$ and let $\sigma_{1,1}=f\left(\xi_{1,1}\right)$. Set $\rho_{1,1}:=$ $d\left(w_{0,1}, \sigma_{1,1}\right)$, where $w_{0,1}=f\left(x_{1,1}\right)$. Then

$$
B\left(w_{0,1}, \rho_{1,1}\right) \cap \Omega \subset f\left(\tilde{Q}\left(b_{1,1}\right)\right) .
$$

So, by Lemma 2.2,

$$
u(f(z)) \leq C \delta^{\alpha k} \max _{\tilde{Q}\left(b_{1,1}\right)} u \circ f
$$

Choose $k$, depending only on $p$, to be the least positive integer such that

$$
C \delta^{\alpha k}<\Lambda^{-1}
$$

This choice of $k$ determines $A$ (say $A=2 \Lambda^{k}$ ) which therefore also depends only on $p$ (since $\delta$ is fixed in (5.3)). With this choice of $A$ we have

$$
\max _{\xi_{1,1}} U>\Lambda U(z)>\Lambda A U(a) .
$$


Since $U\left(b_{1,1}\right) \leq \Lambda U(a)$ we see from (5.8) that we can now repeat the above argument with $\tilde{Q}\left(b_{1,1}\right)$ playing the role of $\tilde{Q}(a)$. That is, we find $b_{1,2}$ on the vertical sides of $\tilde{Q}\left(b_{1,1}\right)$ with $\operatorname{Im} b_{1,2}=\delta_{\star}^{2} \operatorname{Im} a$ and a box $\tilde{Q}\left(b_{1,2}\right)$ with boundary $\xi_{1,2}$ such that

$$
\max _{\xi_{1,2}} U>\Lambda^{2} A U(a) \geq A U\left(b_{1,2}\right)
$$

Continuing by induction we get a contradiction because $U=0$ continuously on $\mathbb{R}$. If $z \in \lambda_{2,1}$, we get a contradiction by the same argument. Thus, there exists $A=A(p) \geq 1$ for which (4.13) holds. The proof of Theorem 2 is now complete.

\section{References}

[AS05] H. Aikawa and N. Shanmugalingam, Carleson-type estimates for p - harmonic functions and the conformal Martin boundary of John domains in metric measure spaces, Michigan Math. J. 53 (2005), 165 -188.

[Ba96] A. Batakis, Harmonic measure of some Cantor type sets, Ann. Acad. Sci. Fenn. Math. 21 (1996), no 2, 255-270.

[BB99] Z. Balogh, and M. Bonk Lengths of radii under conformal maps of the unit disc. Proc. Amer. Math. Soc. 127 (1999), no. 3, 801-804.

[BL05] B. Bennewitz and J. Lewis, On the dimension of p-harmonic measure, Ann. Acad. Sci. Fenn. Math., 30 (2005), no. 2, 459-505.

[Bo87] J. Bourgain, On the Hausdorff dimension of harmonic measure in higher dimensions, Inv. Math. 87 (1987), 477-483.

[C62] L. Carleson, On the existence of boundary values for harmonic functions in several variables, Ark. Mat. 4 (1962), 393-399.

[C85] L. Carleson, On the support of harmonic measure for sets of Cantor type, Ann. Acad. Sci. Fenn. 10 (1985), 113 - 123.

[CFMS81] L. Caffarelli, E. Fabes, S. Mortola, S. Salsa, Boundary behavior of nonnegative solutions of elliptic operators in divergence form, Indiana J. Math. 30 (4) (1981) 621-640.

[D57] Y. Domar, On the existence of a largest subharmonic minorant of a given function, Ark. Mat. 3 (1957), 429-440.

[GM05] J. Garnett and D. Marshall, Harmonic Measure, Cambridge University Press, 2005.

[HK07] H. Hedenmalm and I. Kayamov, On the Makarov law of the iterated logarithm, Proc. Amer. Math. Soc. 135 (2007), no. 7, 2235-2248.

[HKM93] J. Heinonen, T. Kilpeläinen, and O. Martio, Nonlinear potential theory of degenerate elliptic equations, Oxford University Press, 1993. 
[JW88] P. Jones and T. Wolff, Hausdorff dimension of harmonic measure in the plane, Acta Math. 161 (1988), 131-144.

[KW85] R. Kaufmann and J.M. Wu, On the snowflake domain, Ark. Mat. 23 (1985), 177-183.

[L06] J. Lewis, Note on p harmonic measure, Computational Methods in Function Theory 6 (2006), No.1, 109-144.

[LN07] J. Lewis and K. Nyström, Boundary Behaviour for p-Harmonic Functions in Lipschitz and Starlike Lipschitz Ring Domains, Annales Scientifiques de L'Ecole Normale Superieure, Volume 40, Issue 5, September-October 2007, 765-813.

[LN] J. Lewis and K. Nyström, Boundary Behaviour and the Martin Boundary Problem for p-Harmonic Functions in Lipschitz domains, to appear in Annals of Mathematics.

[LN08a] J. Lewis and K. Nyström, Regularity and Free Boundary Regularity for the p-Laplacian in Lipschitz and $C^{1}$-domains, Annales Acad. Sci. Fenn. Mathematica 33 (2008), 523 -548 .

[LN08b] J. Lewis and K. Nyström, Boundary Behaviour of p-Harmonic Functions in Domains Beyond Lipschitz Domains, Advances in the Calculus of Variations 1 (2008), 133 177.

[LVV05] J. Lewis, G. Verchota, and A. Vogel, On Wolff snowflakes, Pacific Journal of Mathematics 218 (2005), 139-166.

[Mak85] N. Makarov, Distortion of boundary sets under conformal mapping, Proc. London Math. Soc. 51 (1985), 369-384.

[Mat95] P. Mattila, Geometry of Sets and Measures in Euclidean Spaces, Cambridge University Press, 1995.

[P75] C. Pommerenke, Univalent Functions, Vandenhoeck and Ruprecht, Göttingen, 1975.

[V93] A. Volberg, On the dimension of harmonic measure of Cantor repellers, Michigan Math. J, 40 (1993), 239-258.

[W93] T. Wolff, Plane harmonic measures live on sets of finite linear length, Ark. Mat. 31 (1993), no. 1, 137-172.

[W95] T. Wolff, Counterexamples with harmonic gradients in $R^{3}$, Essays in honor of Elias M. Stein, Princeton Mathematical Series 42 (1995), 321-384. 